\title{
CONCORDANCIA ENTRE LAS ESCALAS DE RIESGO CARDIOVASCULAR PROCAM Y FRAMINGHAM EN VARONES QUE RECIBEN TRATAMIENTO ANTIRRETROVIRAL EN UN HOSPITAL NACIONAL DE LIMA, PERÚ 2013
}

\author{
Patricia Lister-Del Pino1,a, Gustavo León-Amenero1,a , Angela Leiva-Montejo ${ }^{1, a}$, Eddy R. Segura ${ }^{1, b}$
}

\begin{abstract}
RESUMEN
Objetivos. Determinar la concordancia entre las escalas PROCAM (Prospective Cardiovascular Münster) y Framingham en pacientes receptores de tratamiento antirretroviral. Materiales y métodos. Estudio transversal analítico en población masculina VIH positiva que recibe tratamiento antirretroviral en un hospital de referencia nacional en Lima, Perú. Para evaluar la concordancia entre ambas escalas se empleó el método gráfico de Bland y Altman, correlación con el coeficiente de Pearson y acuerdo entre pruebas mediante el coeficiente de Kappa. Resultados. Enrolamos 111 personas, con edad promedio de 47,0 años. La distribución de pacientes en riesgo cardiovascular bajo, moderado y alto fue de $81,2 \%, 13,6 \%$ y $5,4 \%$, según PROCAM y de $71,2 \%, 25,2 \%$ y 3,6\% según Framingham, respectivamente. Según el método de Bland y Altman, la concordancia fue adecuada en valores bajos y se fue perdiendo en riesgos altos. El coeficiente de Pearson mostró fuerte correlación $(r=0,87$ y $p<0,05)$ y el coeficiente de Kappa fue de 0,56 $(p<0,001)$. Conclusiones. La concordancia existente a riesgos bajos se va perdiendo a medida que el riesgo se va incrementando. Existe fuerte correlación entre ambas escalas. Se recomienda realizar más estudios que permitan conocer qué escala es la más óptima para ser utilizada en población adulta VIH positiva que recibe tratamiento antirretroviral.
\end{abstract}

Palabras clave: Medición de riesgo; ARV; Estudio comparativo; Enfermedades cardiovasculares (fuente: DeCS BIREME).

\section{CONCORDANCE BETWEEN PROCAM AND FRAMINGHAM CARDIOVASCULAR RISK SCORES AMONG MEN RECEIVING HIV TREATMENT AT A NATIONAL HOSPITAL IN LIMA, PERU 2013}

\begin{abstract}
Objectives. The aim of the study is to determine the concordance between the PROCAM (Prospective Cardiovascular Münster) and Framingham scales in patients receiving highly active antiretroviral therapy (HAART). Materials and methods. A cross sectional study was conducted in HIV-positive male population who use HAART in a national reference hospital located in Lima, Peru. To evaluate the concordance between the two scales the graphic method of Bland and Altman was used, for the evaluation of the correlation we used the Pearson coefficient and to measure the agreement we use the kappa coefficient. Results. 111 patients were enrolled, with an average age of 47.0 years.-The distribution of patients according to the risk was low, moderate and high, $81.2 \%, 13.6 \%$ and $5.4 \%$ respectively for PROCAM and $71.2 \%$, $25.2 \%$ and $3.6 \%$ for Framingham. According to the graphic method of Bland and Altman, the concordance was adequate in low values and was lost as the risk score increased. Pearson's test found a strong correlation $(r=0.87$ and $p<0.05)$ and the kappa coefficient was $0.56(p<0,001)$. Conclusions. The agreement we found at low risk decreases as the risk increases. Strong correlation was found between the two scales. We recommend further studies in order to know which scale of cardiovascular risk is the most optimal scale for clinical practice among HIV population who receive HAART.
\end{abstract}

Key words: Risk assessment, HAART; Comparative study; Cardiovascular diseases (source: MeSH NLM).

Escuela de Medicina, Universidad Peruana de Ciencias Aplicadas. Lima, Perú.

Bachiller de medicina; ${ }^{\mathrm{b}}$ médico cirujano

Los autores declaramos que la presente investigación ha sido presentada como tesis para obtener el título de médico cirujano

Recibido: 19-02-15 Aprobado: 05-08-15

Citar como: Lister-Del Pino P, Gustavo León-Amenero G, Leiva-Montejo A, Segura ER. Concordancia entre las escalas de riesgo cardiovascular PROCAM y FRAMINGHAM en varones que reciben tratamiento antirretroviral en un hospital nacional de Lima, Perú 2013. Rev Peru Med Exp Salud Publica. 2015;32(4):731-8 


\section{INTRODUCCIÓN}

La epidemia causada por el virus de la inmunodeficiencia humana (VIH) tuvo, para el año 2012, 35,3 millones de personas infectadas, con 2,3 millones de casos nuevos a nivel mundial (1). En el Perú, hasta febrero de 2014, el MINSA reportó 52752 casos, además de 308 casos nuevos de los cuales 124 correspondieron a Lima (2). Para su tratamiento se estableció, a nivel mundial, el tratamiento antirretroviral (TAR). En el Perú, en el año 2004, se instauró el TAR como norma ministerial para los centros de salud del estado ${ }^{(3)}$.

Con el inicio del TAR, se realizaron múltiples estudios para determinar sus efectos adversos. Según el estudio DAD (Data Colection on Adverse Events of Anti-HIV Drugs Study), realizado en el 2003, se evidenció que los inhibidores de las proteasas utilizados junto con los inhibidores de la transcriptasa inversa no análogos de nucleósidos, produjeron incremento en la serie de triglicéridos, colesterol, y descenso del c-HDL en los pacientes tratados con dichos esquemas durante cuatro años ${ }^{(4)}$. En el hospital de Massachusetts, EE.UU., se halló un riesgo relativo de 1,75 de sufrir infarto agudo de miocardio para pacientes $\mathrm{VIH}$ en comparación con pacientes no $\mathrm{VIH}{ }^{(5)}$. El estudio HOPS, realizado entre los años 1992 y 2003, encontró un incremento de la frecuencia de los infartos agudos de miocardio desde la aparición del TAR ${ }^{(6)}$. Un estudio en Nigeria, 2013, encontró asociación entre el TAR e hipertensión arterial, obesidad y síndrome metabólico ${ }^{(7)}$.

La presencia de enfermedad cardiovascular conllevó al uso escalas que evalúan el riesgo de desarrollar un evento cardiovascular, entre ellas Framingham y PROCAM. La escala Framingham fue elaborada en el año 2000, en base al estudio Framingham original de EE.UU. que siguió a 5209 varones de 30 a 62 años en dos ocasiones: 1948 y 1971. Esta incluye edad, colesterol total, presión arterial sistólica, tabaco y HDL-c, además es usada en la mayoría de estudios integrados por pacientes VIH positivos. La escala PROCAM fue elaborada en el año 2002, en base al estudio "Prospective Cardiovascular Münster" en Alemania, que siguió a 5389 pacientes de 35 a 65 años, entre 1979 y $1985^{(8,9)}$. Esta incluye edad, LDL-c, HDL-c, triglicéridos, tabaco, diabetes, presión arterial sistólica y antecedente familiar de infarto agudo de miocardio en menores de 60 años ${ }^{(8,9)}$.

La escala Framingham ha sido recomendada por diversas guías como la Adult AIDS Clinical Trial Group Cardiovascular Disease Focus Group (AACTG) ${ }^{(10)}$ y el Consenso de Pavia (11). Este último indicó que todo paciente usuario de TAR requiere una estratificación de riesgo cardiovascular mediante la escala de
Framingham. Por otro lado, algunos especialistas recomendaron utilizar escalas que incluyan otros parámetros no admitidos en la escala Framingham, ya que esta podía subestimar el riesgo cardiovascular (12). Es por ello que en el presente estudio se incluyó la escala PROCAM que cuenta, entre sus variables, con el valor de los triglicéridos, la presencia de diabetes mellitus y el antecedente familiar de infarto agudo de miocardio. Cabe resaltar que la hipertrigliceridemia, medida solo por PROCAM, y los niveles bajos de HDL-c, son signos que generalmente se encuentran alterados dentro de la población $\mathrm{VIH}$ positivaa ${ }^{(13)}$.

Para este estudio no fue posible hacer un seguimiento de diez años de ambas escalas pero sí se podría demostrar si existe concordancia o no. De existir baja concordancia se deberían realizar más estudios para demostrar cuál de las dos brinda el diagnóstico más certero. De existir buena concordancia el médico podría utilizar cualquiera de las dos escalas indiferentemente, ya que los datos faltantes como el antecedente familiar, diabetes mellitus e hipertrigliceridemia no alterarían el resultado del riesgo a diez años. En base a lo expuesto $y$, debido a que existen escasos estudios de este tipo en dicha población, y ninguno realizado en nuestro país, resulta pertinente comparar ambas escalas en pacientes usuarios de TAR.

El objetivo principal del estudio fue determinar la concordancia entre las escalas Framingham y PROCAM de los pacientes varones con VIH usuarios de TAR, que asisten a un hospital nacional en Lima, Perú. Los objetivos secundarios fueron explorar la correlación de las mismas escalas, el valor global de riesgo en la población, y el perfil lipídico.

\section{MATERIALES Y MÉTODOS}

\section{DISEÑO Y LUGAR DE ESTUDIO}

Se planteó un diseño de estudio transversal y analítico para evaluar la concordancia de dos escalas de riesgo cardiovascular en población $\mathrm{VIH}+$ que recibe TAR. Se reclutó a pacientes $\mathrm{VIH}+$ receptores de TAR entre los meses enero a diciembre de 2013 en los consultorios externos del Servicio de Infectología del Hospital Nacional Edgardo Rebagliati Martins (HNERM).

\section{POBLACIÓN}

La población incluida en el estudio correspondió a pacientes con VIH positivo que cumplían con los siguientes criterios de inclusión: varones receptores de TAR por un mínimo de 1 año; en un rango de edad de 35 a 65 años; y que acudieron a consulta externa 
del HNERM entre los meses de enero y diciembre del año 2013. Como criterio de exclusión se consideró a los pacientes con historia de angina de pecho definida por el cuestionario Rose, historia de infarto agudo de miocardio, enfermedad cerebrovascular y enfermedades que alteren el perfil lipídico como hipotiroidismo, síndrome nefrótico, cirrosis y pancreatitis.

\section{VARIABLES}

Las variables principales del estudio fueron los porcentajes de riesgo de las escalas PROCAM y Framingham. Ambos fueron medidos de modo cuantitativo continuo (porcentaje de riesgo de evento cardiovascular en 10 años) y categórico (bajo, menor a $10 \%$; moderado, menor de $20 \%$; alto, mayor o igual de $20 \%$ ). Las variables secundarias fueron las incluidas en dichas escalas (edad, tabaquismo, presión arterial sistólica, diabetes mellitus, antecedente familiar de infarto de miocardio agudo en menores de 60 años, colesterol HDL, LDL y total, y triglicéridos), además de peso, talla e índice de masa corporal. La variable "dislipidemia" se tomó como elevación de colesterol total $\geq 200 \mathrm{mg} / \mathrm{dL}$ y/o LDL $\geq 160 \mathrm{mg} / \mathrm{dL}$ y/o triglicéridos $\geq 200$ $\mathrm{mg} / \mathrm{dL}$ según guía ATP-III ${ }^{(14)}$ y la guía de la "American Association of Clinical Endocrinologist" (15).

El estudio no presentó variables de exposición ni de respuesta ya que se buscó concordancia entre dos escalas.

\section{FUENTE DE DATOS/MEDICIÓN}

Enrolamos a los participantes en la consulta externa del servicio de infectología, previa coordinación con los médicos asistentes. Ellos tuvieron el primer contacto con los pacientes a quienes invitaron a participar en el estudio. Las invitaciones fueron en forma consecutiva, es decir, en forma no probabilística. Después de aceptar, los pacientes se reunieron con los investigadores para el recojo de datos. El médico asistente se encargó de facilitar las respectivas historias clínicas para la extracción de datos de laboratorio.

Se empleó un instrumento de recolección de datos elaborado por los investigadores en el cuál se incluyeron las variables: edad, peso, talla, antecedente familiar de infarto agudo de miocardio en menores de 60 años, diabetes mellitus, tabaquismo y presión arterial, obtenidos mediante la entrevista, y colesterol LDL, HDL y total, y triglicéridos, de la historia clínica, con un tiempo de antigüedad no mayor a tres meses al enrolamiento en el estudio. Para preservar la identidad de los pacientes los datos recolectados fueron anotados con un código, el cual fue también entregado al paciente para que recoja su resultado al finalizar el estudio.
No se recolectó información respecto al esquema TAR de cada paciente, debido a la constante variación de los fármacos retrovirales por factores externos como la disponibilidad de los mismos.

El riesgo cardiovascular para cada escala fue medido de acuerdo a las tablas de medición para cada una de las escalas. Estas tablas se obtuvieron de los estudios PROCAM $^{(8)}$ y Framingham ${ }^{(9)}$.

\section{TAMAÑO MUESTRAL}

El cálculo de tamaño muestral no fue realizado para el método gráfico de Bland y Altman sino para un coeficiente rho de Pearson esperado de $+0,86$. Ello, debido a que el coeficiente rho de Pearson era el más comúnmente reportado ${ }^{(16)}$. Asumiendo una amplitud del intervalo de confianza del coeficiente de rho de 0,10 unidades, resultaba en un mínimo de 111 sujetos por analizar. Considerando una tasa de rechazo del 20\%, requeríamos invitar a 150 pacientes. Para realizar los cálculos descritos, se trabajó con el programa PASS $®$.

\section{ANÁLISIS ESTADÍSTICO}

Se empleó el programa Stata versión 11 para Windows para el análisis estadístico y Microsoft Excel 2010® para la digitación de la información. Se empleó el test de shapiro wilk para evaluar la distribución de las variables cuantitativas. Estas fueron presentadas como media y desviación estándar o como mediana y rango intercuartílico de acuerdo a su distribución, y las variables cualitativas como porcentajes. Además, se determinó la correlación mediante el test de correlación de Pearson, ello para satisfacer un objetivo secundario.

El análisis principal fue determinar la concordancia entre ambas escalas, para ello se empleó el modelo gráfico de Bland y Altman. Este método de Bland y Altman es un método gráfico para la estimación de la concordancia entre dos mediciones repetidas a un mismo individuo. Este representa mediante un gráfico de dispersión la media de dos mediciones frente a la diferencia absoluta de las mismas. El gráfico tiene una línea central que representa la diferencia media y dos horizontales marginales que son los límites de concordancia. Si a lo largo de la medición los valores son estables y siguen un patrón normal, 95\% de los valores serán concordantes ${ }^{(17)}$. También, para determinar la correlación se utilizó el coeficiente rho de Pearson, y calculamos el acuerdo entre las escalas categorizadas mediante el coeficiente de Kappa.

\section{CONSIDERACIONES ÉTICAS}

El presente estudio fue aprobado por el comité de ética de la Universidad Peruana de Ciencias Aplicadas y por 
el Comité de Capacitación del HNERM. Se contó con el consentimiento informado por escrito debido a que se trató con población potencialmente vulnerable.

\section{RESULTADOS}

Debido a que el médico tratante era el que aplicaba los criterios de inclusión, no se tuvo acceso al número de examinados para elegibilidad. Sin embargo, los elegibles confirmados fueron 134 , de los cuales se excluyeron 23 (13 por no cumplir con criterio "edad", 4 por haber iniciado TAR en los últimos 12 meses y 6 por no tener el perfil de laboratorio completo). Finalmente, se contó con 111 pacientes incluidos en el estudio (Figura 1).

Se presenta la descripción de variables numéricas y categóricas que pertenecen a ambas escalas. Cabe resaltar los datos de mayor implicancia del estudio. En cuanto a las variables numéricas, la mediana de la edad fue de 47 años; la media de IMC, triglicéridos y colesterol fue de $25,4 \mathrm{~kg} / \mathrm{m}^{2}, 265,0 \mathrm{mg} /$ $\mathrm{dL}$ y $231,8 \mathrm{mg} / \mathrm{dL}$, respectivamente. En cuanto a las variables categóricas, las más resaltantes fueron el tabaquismo presente en 20 pacientes (18\%) y el resultado de PROCAM "bajo" y Framingham "bajo", correspondientes a un $90(81,2 \%)$ y $79(71,2 \%)$, respectivamente. No se evidenciaron datos faltantes para cada variable de interés en la población estudiada. Las características principales de los participantes del estudio se muestran en la Tabla 1.

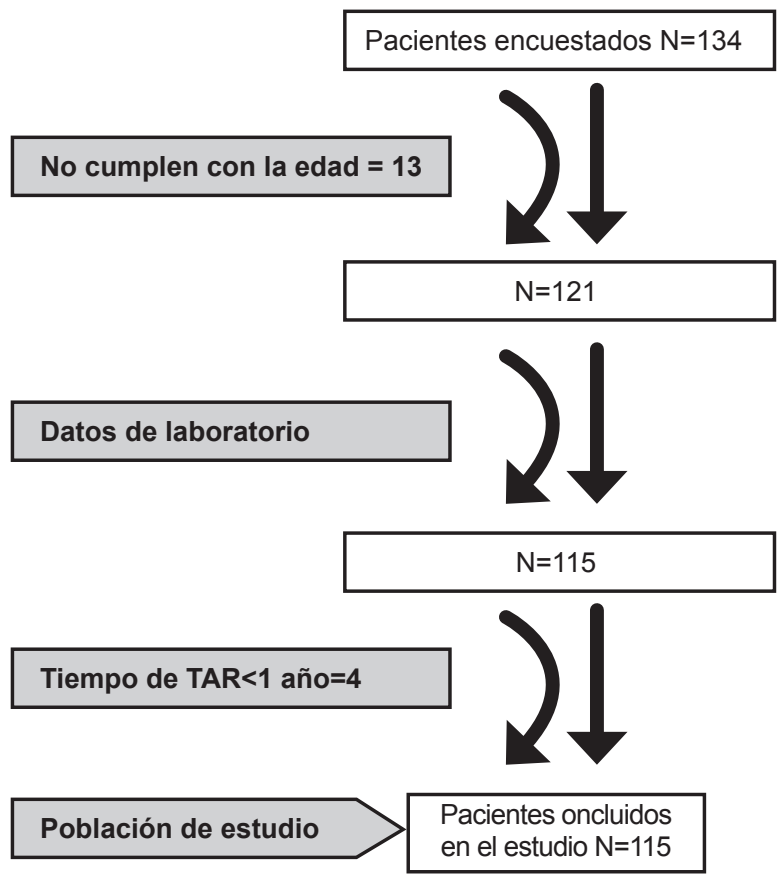

Figura 1. Flujograma de selección de participantes
Tabla 1. Características Generales de Población estudiada $(n=111)$

\begin{tabular}{|c|c|c|}
\hline Características & $\mathbf{n}$ & $\begin{array}{l}\text { Medida dispersión/ } \\
\text { Frecuencia relativa }\end{array}$ \\
\hline Edad (años) ${ }^{* *}$ & 47 & RIC: $43-53$ \\
\hline Peso $(\mathrm{kg})^{*}$ & 73,8 & DE: 10.62 \\
\hline Talla $(m)^{*}$ & 1,69 & DE: 0,08 \\
\hline Índice de masa corporal $\left(\mathrm{kg} / \mathrm{m}^{2}\right)^{*}$ & 25,39 & DE: 3.90 \\
\hline $\begin{array}{l}\text { Presión arterial sistólica } \\
(\mathrm{mmHg})^{*}\end{array}$ & 120,52 & DE: 13,83 \\
\hline $\begin{array}{l}\text { Presión arterial diastólica } \\
(\mathrm{mmHg})^{\star}\end{array}$ & 79,6 & DE: 9,40 \\
\hline Tabaquismo*** & 20 & $18 \%$ \\
\hline Diabetes mellitus*** & 2 & $1,8 \%$ \\
\hline Dislipidemia*** & 85 & $76,6 \%$ \\
\hline $\begin{array}{l}\text { Antecedente familiar de infarto } \\
\text { agudo de miocardio*** }\end{array}$ & 12 & $10,8 \%$ \\
\hline $\begin{array}{l}\text { Lipoproteínas de baja densidad } \\
(\mathrm{mg} / \mathrm{dL})^{*}\end{array}$ & 136,9 & DE: 46,8 \\
\hline $\begin{array}{l}\text { Lipoproteínas de alta densidad } \\
(\mathrm{mg} / \mathrm{dL})^{*}\end{array}$ & 47,2 & DE: 18,9 \\
\hline Triglicéridos $(\mathrm{mg} / \mathrm{dL})^{*}$ & 265,0 & DE: 213,8 \\
\hline Colesterol $(\mathrm{mg} / \mathrm{dL})^{*}$ & 231,8 & DE: 107,8 \\
\hline Hipertrigliceridemia*** & 59 & $53,2 \%$ \\
\hline Hipercolesterolemia*** & 69 & $62,2 \%$ \\
\hline PROCAM bajo*** & 90 & $81,2 \%$ \\
\hline PROCAM moderado*** & 15 & $13,6 \%$ \\
\hline PROCAM alto*** & 6 & $5,41 \%$ \\
\hline Framingham bajo*** & 79 & $71,2 \%$ \\
\hline Framingham moderado*** & 28 & $25,2 \%$ \\
\hline Framingham alto*** & 4 & $3,6 \%$ \\
\hline
\end{tabular}

Finalmente, se encontró una frecuencia absoluta de 85 pacientes $(76,6 \%)$ con dislipidemia. De ellos $81,2 \%$ presentaron colesterol total $\geq 200 \mathrm{mg} / \mathrm{dL}, 69,4 \%$ triglicéridos $\geq 200 \mathrm{mg} / \mathrm{dL}$ y $44,7 \% \mathrm{LDL} \geq 160 \mathrm{mg} / \mathrm{dL}$. Estos criterios se detectaron en $20,7 \%$ de la muestra.

Mediante el método gráfico de Bland y Altman se observó que la concordancia existente a niveles bajos de riesgo cardiovascular se pierde a medida que el riesgo cardiovascular se incrementa (Figura 2). El coeficiente de Kappa fue de 0,56 ( $p<0,001$ ) (IC 95\%: 0,41-0,71). Para las escalas, el coeficiente de correlación de Pearson fue 0,87 ( $<<0,001)$ (Figura 3).

La distribución del riesgo según cada score se puede observar en la Tabla 1. Por otro lado, se determinó un valor global de riesgo de 7,2\% (DE=6,4\%) para Framingham y de 5,9\% (DE = 7,1\%) para PROCAM.

Según la distribución de pacientes por riesgos según cada escala (Tabla 2 ), el $85,6 \%$ de pacientes catalogados como "bajo riesgo" para PROCAM, también lo fueron para Framingham. El 50,0\% de los pacientes catalogados como "alto riesgo" para PROCAM también lo fueron para Framingham, el resto fue riesgo moderado. Según el score Framingham, se 


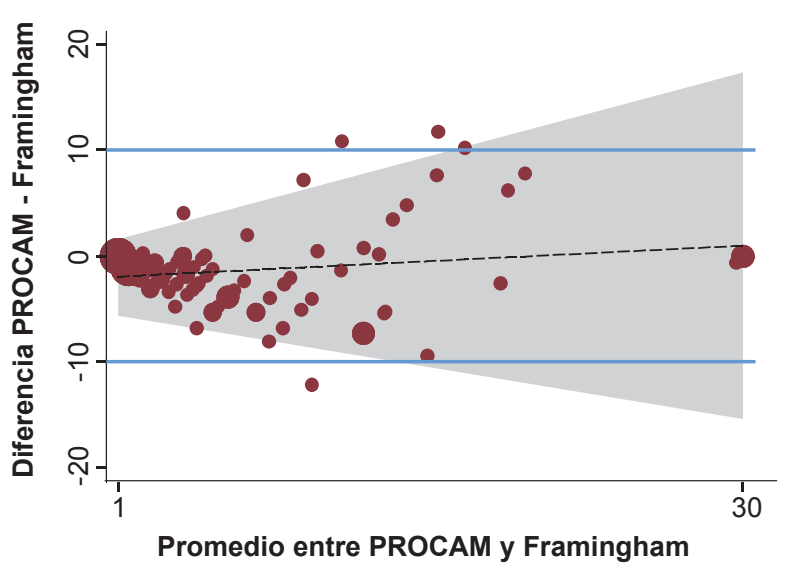

Línea punteada: diferencia media.

Las líneas azules: indican los límites de concordancia (+/- 2 DE de la media).

Figura 2. Gráfico de Bland y Altman para determinar concordancia entre los scores PROCAM (Prospective Cardiovascular Münster) y Framingham

encontraron cuatro pacientes de "alto riesgo"; de ellos, PROCAM cataloga a tres como "alto riesgo" y uno como "moderado riesgo".

\section{DISCUSIÓN}

La concordancia determinada por el estudio según la evaluación del gráfico de Bland y Altman fue adecuada en valores bajos y se fue perdiendo mientras el score de riesgo fue incrementándose. Dicho resultado concuerda con un estudio en población no VIH realizado en España (16) donde determinaron que la concordancia disminuye a medida que se incrementa el riesgo de acuerdo a las escalas. Asimismo, mediante la evidencia gráfica del método Bland y Altman, el PROCAM sobreestima los altos riesgos y subestima los bajos riesgos, dato similar a nuestro hallazgo. Es importante resaltar que nuestro estudio es el único encontrado en la bibliografía que utiliza el método gráfico Bland y Altman en población receptora de TAR.
El coeficiente de Kappa determinado, aunque fue significativo $(p<0,001)$, tuvo un valor de 0,56 que equivale a una concordancia moderada. Ello es muy similar al hallado en un estudio hecho en Brasil (18) $y$ otro en España ${ }^{(19)}$ con $\mathrm{K}=0,65$ y $\mathrm{K}=0,36$ respectivamente. Se determinó el Kappa por brindar un valor numérico para la estimación de la concordancia, haciéndola más evaluable, a diferencia del Bland y Altman que muestra resultados solo gráficos; sin embargo, se priorizan los resultados de este último ya que la naturaleza de la fórmula de Kappa solo está preparada para valorar escalas en modo cualitativo.

Con los resultados obtenidos se determina que las escalas no son suficientemente concordantes para poder ser utilizadas indiferentemente ni reemplazables una con la otra. No obstante, no se conoce cuál da un riesgo más cercano al real pues para ello se requiere de un seguimiento poblacional a diez años y mayor muestra. Se debe recalcar que la menor concordancia se presenta en los grupos de alto riesgo lo cual es importante, debido a que esta es la población que requiere mayor precisión predictiva para las medidas terapéuticas.

Consideramos que la no completa concordancia encontrada entre ambas escalas, así como el mayor riesgo determinado por la escala PROCAM, puede ser explicado por la inclusión de las variables triglicéridos, antecedente familiar de infarto de miocardio y riesgo global poblacional de donde se desarrolló cada una de las escalas.

La correlación hallada mediante Pearson determinó un valor de $0,87(p<0,001)$ siendo catalogada como "fuerte". Este resultado es similar al presentado por Álvarez et al. con un coeficiente de correlación de Pearson de $0,86^{\left({ }^{(16)}\right.}$. La buena correlación encontrada en ambos estudios es atribuida a la escasa diferencia en cuanto a las variables tomadas por ambas escalas. Si bien la correlación no sirve para demostrar concordancia, ha sido empleada para satisfacer un objetivo secundario. Este fue planteado para mostrar el contraste entre el mensaje dado entre el gráfico de Bland y Altman, y la

Tabla 2. Distribución de pacientes según riesgos de escalas PROCAM (Prospective Cardiovascular Münster) Y Framingham

\begin{tabular}{|c|c|c|c|c|c|}
\hline & & \multicolumn{3}{|c|}{ Framingham } & \multirow{2}{*}{ Total (\%) } \\
\hline & & Bajo riesgo (\%) & Riesgo moderado (\%) & Alto riesgo (\%) & \\
\hline \multirow{4}{*}{ 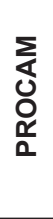 } & Bajo riesgo (\%) & $77(85,6)$ & $13(14,4)$ & $0(0)$ & $90(100)$ \\
\hline & Riesgo moderado (\%) & $2(13,3)$ & $12(80)$ & $1(6,7)$ & $15(100)$ \\
\hline & Alto riesgo (\%) & $0(0)$ & $3(50)$ & $3(50)$ & $6(100)$ \\
\hline & Total (\%) & $79(71,2)$ & $28(25,2)$ & $4(3,6)$ & $111(100)$ \\
\hline
\end{tabular}


correlación de Pearson. Además, permite ampliar la comparación de nuestro hallazgo con otros estudios, pues es el método más empleado.

Seevidenció queamayor riesgo, elPROCAMsobreestima los resultados en comparación al Framingham; en otras palabras, el Framingham infravalora los resultados con respecto al PROCAM (Figura 2).

En estudios realizados en Brasil, España, Australia y en un análisis del DAD (Law MG et al.) (16, 18-21), así como en el presente estudio, la mayoría de pacientes (>45\%) fueron catalogados dentro del grupo de bajo riesgo. El patrón de riesgos, así como en los otros estudios, tuvo una distribución piramidal siendo la base el grupo de bajo riesgo, seguido por el de riesgo moderado y alto.

La prevalencia encontrada de riesgo moderado y alto para Framingham fue de $21,4 \%$, muy superior al $9 \%$ descrito por un reporte del estudio DAD elaborado por Law MG et al. ${ }^{(20)}$, e inferior al $29,1 \%$ encontrado en un estudio local de Australia, Hadigan C. et al., quien también trabajó con población VIH receptora de TAR ${ }^{(21)}$. No se ha encontrado bibliografía que respalde dicha diferencia, esta se atribuye a factores raciales y/o a una muestra insuficiente.

Un 76,6\% de la muestra tuvo dislipidemia, la cual es mayor al $42 \%$ encontrado en el estudio nacional de Villegas et al. ${ }^{22}$. La frecuencia relativa de hipertrigliceridemia fue $53,2 \%$, muy superior al $28 \%$ del estudio de Villegas et al., y similar al $52 \%$ de un estudio arequipeño ${ }^{(23)}$. Un estudio Francés encontró $28 \%$ de hipertrigliceridemia (inferior al $53,2 \%$ hallado) ${ }^{(24)}$. Villegas en Perú, y Mulligan K. y Worm S.W. en EE.UU. confirman la vulnerabilidad de los pacientes receptores de TAR a la alteración del perfil lipídico, independientemente de los factores culturales y temporales ${ }^{(13,22,25)}$. Se especula que la diferencia hallada podría ser atribuida al diferente régimen dietético entre las culturas, a la variación de puntos de corte para la definición de hipertrigliceridemia e hipercolesterolemia en los diferentes estudios, o al abordaje únicamente a varones que tuvo el presente estudio.

En el 2013, la "American College of Cardiology" (ACC), en asociación con la "American Heart Asociation" (AHA) publicó el "Guideline on the Assessment of Cardiovascular Risk" (26) y el "Guideline on the treatment of blood cholesterol to reduce artherosclerotic cardiovascular risk in adults: a report of the American College of Cardiology/ American Heart Association Task Force on practice guidelines" (27). En ellas se promueve el empleo de la escala "ASCVD-Risk", la cual no fue empleada por no contar con la variable "triglicéridos"; además, debido a la reciente aparición de la misma, sus resultados no han podido ser evidenciados hasta la fecha.
El estudio cuenta con ciertas limitaciones como la reducida aplicabilidad de las estimaciones de riesgo a las poblaciones diferentes de las que se derivaron, debido a que cualquier sistema de puntuación de riesgo cardiovascular primero debe ser evaluado a fondo antes de su introducción y necesita ser actualizada basándose en las tendencias de cambio de los factores de riesgo. Además, existe un sesgo de selección debido a que se accedió a la muestra de acuerdo al orden de los pacientes citados, que cumplieran con los criterios de inclusión y aceptaran participar; consideramos que este sesgo es mínimo ya que el orden de la consulta no está sujeto a ninguna variable demográfica, clínica ni terapéutica de la enfermedad. Del mismo modo, el estudio no incluye mujeres debido a que la escala PROCAM solo es aplicada en varones. Otra limitación corresponde al número de pacientes dentro del grupo de alto riesgo, ya que al ser pequeño está sujeto a un nivel de error amplio. Por último, la proporción de pacientes con dislipidemia no son necesariamente representativos por el reducido tamaño muestral.

Podemos concluir que la concordancia existente a riesgos bajos se va perdiendo a medida que el riesgo va incrementando. La escala PROCAM incluye más pacientes de la muestra dentro del grupo de alto riesgo en comparación a la escala Framingham. Además, se halló una buena correlación y regular acuerdo entre ambas escalas de riesgo cardiovascular.

Recomendamos la elaboración de un estudio de validación para ambas escalas a nivel local, es decir, contrastar los riesgos estimados por estas escalas contra los eventos cardiovasculares que sucedan a 10 años. Asimismo, se recomienda que en estudios similares realizados a futuro se incluyan variables como el tratamiento y su relación con la dislipidemia (hiperlipemia, hipertrigliceridemia, hipercolesterolemia); además, del estudio de otras variables como el valor de linfocitos T CD4 y la proporción de pacientes con carga viral detectable ya que estos pueden presentar elevación de triglicéridos o descenso de HDL. Finalmente, en nuestro medio debería ser frecuente la valoración integral del paciente en TAR mediante una evaluación de su riesgo cardiovascular con el uso de algunas de las escalas estudiadas.

Contribuciones de autoría: los autores han contribuido en la concepción y diseño del artículo, la recolección de datos, redacción y aprobación de la versión final. GLA y ERS realizaron el análisis e interpretación de datos.

Fuentes de financiamiento: el presente estudio fue autofinanciado.

Conflictos de interés: el presente estudio no presenta conflictos de interés. 


\section{REFERENCIAS BIBLIOGRÁFICAS}

1. Programa Conjunto de las Naciones Unidas sobre el $\mathrm{VIH} /$ sida (ONUSIDA). Informe mundial: ONUSIDA, informe sobre la epidemia mundial de sida 2013. México, D.F.: ONUSIDA; 2013.

2. Dirección General de Epidemiología, Ministerio de Salud del Perú. Situación del VIH/SIDA en el Perú. Boletín Epidemiológico Mensual [Internet]. Febrero 2014 [citado el 5 de febrero de 2015]. Disponible en: http://www. dge.gob.pe/portal/docs/vigilancia/ vih/Boletin_2014/febrero.pdf

3. División General de Salud de las Personas, Ministerio de Salud del Perú. Norma Técnica para el Tratamiento Antirretroviral de Gran Actividad -TARGA en Adultos infectados por el Virus de la Inmunodeficiencia Humana. Lima: MINSA; 2004.

4. Friis-Moller N, Weber R, Reiss $P$, Thiébaut R, Kirk O, d'Arminio Monforte A, et al. Cardiovascular disease risk factors in HIV patients: association with antiretroviral therapy. Results from the DAD Study. AIDS. 2003; 17(8):1179-93.

5. Triant VA, Lee $H$, Hadigan C, Grinspoon SK. Increased acute myocardial infarction rates and cardiovascular risk factors among patients with Human immunodeficiency virus. J Clin Endocrinol Metab. 2007;92(7):25062512.

6. Holmberg SD, Moorman AC, Williamson JM, Tong TC, Ward DJ, Wood KC, et al. HIV Outpatient Study (HOPS) investigators. Protease Inhibitor and cardiovascular outcomes in patients with HIV-1. Lancet. 2002;360(9347):1747-8.

7. Muhammad S, Sani MU, Okeahialam BN. Cardiovascular disease risk factors among HIV-infected Nigerians receiving highly active antiretroviral therapy. Niger Med J. 2013;54(3):18590. doi: 10.4103/0300-1652.114591.

8. Assamann G, Cullen P, Schulte H. Simple scoring scheme for calculating the risk of acute coronary events based on the 10-year follow-up of the prospective cardiovascular Münster (PROCAM) Study. Circulation. 2002;105:310-5.
9. D’Agostino RB, Russell MW, Huse DM, Ellison RC, Silbershatz H, Wilson PW, et al. Primary and subsequent coronary risk appraisal: new results from the Framingham study. Am Heart J. 2000;139(2 Pt 1):272-81.

10. Dubé MP, Stein JH, Aberg JA, FIchtenbaum CJ, Gerbe JG, Tashima $\mathrm{KT}$, et al. Guidelines for the evaluation and management of dyslipidemia in human immunodeficiency virus (HIV)-infected adults receiving antiretroviral therapy: recommendations of the HIV Medical Association of the Infectious Disease Society of America and the Adult AIDS Clinical Trials Group. Clin Infect Dis. 2003;37(5):613-27.

11. Volberding PA, Murphy RL, Barbaro G, Barbarini G, Bruno R, Cirelli A, et al. The Pavia consensus statement. AIDS 2003;17 Suppl 1:S170-9.

12. Hsue PY, Squires K, Bolger AF, Capili B, Mensah GA, Temesgen Z, et al. Screening and Assessment of Coronary Heart Disease in HIV-Infected Patients. Circulation. 2008;118(2):e41$7 . \quad$ doi: 10.1161/ CIRCULATIONAHA.107.189626.

13. Worm SW, Sabin C, Weber R, Reiss P, El-Sadr W, Dabis F, et al. Risk of myocardial infarction in patients with HIV infection exposed to specific individual antiretroviral drugs from the 3 major drug classes: the data collection on adverse events of antiHIV drugs (D:A:D) study. J Infects Dis. 2010;201(3):318-30. doi: 10.1086/649897.

14. National Cholesterol Education Program (NCEP) Expert Panel on Detection, Evaluation and Treatment of High Blood Cholesterol in Adults (Adult Treatment Panel III). Third Report of the National Cholesterol Education Program (NCEP) Expert Panel on Detection, Evaluation and Treatment of High Blood Cholesterol in Adults (Adult Treatment Panel III) final report. Circulation. 2002;106(25):3143-421.

15. Jellinger PS, Smith DA, Mehta AE, Ganda O, Handelsman Y, Rodbard $\mathrm{HW}$, et al. American Association of Clinical Endocrinologists' Guidelines for Management of Dyslipidemia and
Prevention of Atherosclerosis. Endocr Pract. 2012;18(Suppl 1):1-78.

16. Álvarez-Cosmea A, López-Fernández V, Prieto-Díaz MA, Díaz-González L, Herrero-Puente P, Vázquez-Álvarez $\mathrm{J}$, et al. PROCAM y Framingham por categorías: ¿miden igual riesgo? Medifam. 2002;12(4):40-9.

17. Consellería de Sanidade, Xunta de Galicia. Epidad 4: Ayuda de concordancia y consistencia [internet]. Santiago de Compostela, España: Xunta de Galicia; 2014 [citado el 25 de marzo de 2015]. Disponible en: https://www.sergas. es/gal/documentacionTecnica/docs/ SaudePublica/Apli/Epidat4/Ayuda/ Ayuda_Epidat4_Concordancia_y_ consistencia_Octubre2014.pdf

18. Barros ZM, de Alencar Ximenes RA, Miranda-Filho DB, de Albuquerque Mde F, Melo HR, Carvalho EH, et al. Comparison between the Framingham and Prospective cardiovascular of Münster scores for risk assessment of coronary heart disease in human immunodeficiency virus-positive patients in Pernambuco, Brazil. Metab Syndr Relat Disord. 2010;8(6):489-97. doi: 10.1089/met.2009.0100.

19. Knobel H, Jericó C, Montero M, Sorli ML, Velat M, Guelar A, et al. Global cardiovascular risk in patients with HIV Infection: concordance and differences in estimates according to three risk equations (Framingham, SCORE, and PROCAM). AIDS Patient Care STDS. 2007;21(7):452-7.

20. Law MG, Friis-Moller N, El-Sadr WM, Weber R, Reiss P, D’Arminio Monforte $\mathrm{A}$, et al. The use of the Framingham equation to predict myocardial infarctions in HIV-infected patients: comparison with observed events in the D:A:D Study. HIV Med. 2006;7(4):218-30.

21. Hadigan C, Meigs JB, Wilson PW, D'Agostino RB, Davis B, Basgoz N, et al. Prediction of coronary heart disease risk in HIV-infected patients with fat redistribution. Clin Infects Dis, 2003;36(7):909-16.

22. Villegas-Chiroque M, MezarinaValverde JE. Dislipidemia durante la terapia antirretroviral en pacientes con infección por VIH/SIDA atendidos 
en el HNERM, 200-2003 [Tesis de especialidad]. Lima: Facultad de Medicina Humana, UNMSM; 2004. Disponible en: http://sisbib.unmsm. edu.pe/bibvirtual/monografias/salud/ villegas_chm/contenido.htm

23. Valencia-Arroyo BM, TaramonaEspinoza CP, Manrique-Hurtado H. Estudio piloto de las alteraciones metabólicas y síndrome metabólico inducidas por la terapia antirretroviral en pacientes con VIH del Hospital Nacional Arzobispo Loayza, Lima, Perú. Acta Med Peruana. 2008;25(3):153-6.

24. Savés M, Raffi F, Capeau J, Rozenbaum W, Ragnaud JM, Perronne C, et al. Factors related to lipodystrophy and metabolic alterations in patients with human immunodeficiency virus infection receiving highly active antiretroviral therapy. Clin Infect Dis. 2002;34(10)1396-405.

25. Mulligan K, Grunfeld C, Tai VW, Algren H, Pang M, Chernoff DN, et al. Hyperlipidemia and insulin resistance are induced by protease inhibitors independent of changes in body composition in patients with HIV infection. J Acquir Immune Defic Sydr. 2000;23(1):35-43.

26. Goff DC Jr, Lloyd-Jones DM, Bennett G, Coady S, D’Agostino RB, Gibbons $\mathrm{R}$, et al.2013 ACC/AHA guideline on the assessment of Cardiovascular Risk: A Report of the American College of Cardiology/American Heart Association Task Force on Practice
Guidelines. Circulation. 2014;129(25

Suppl 2):S74-5. doi: 10.1161/01. cir.0000437741.48606.98.

27. Stone N, Robinson J, Lichtenstein A, Bairey CN, Blum CB, Eckel R, et al. 2013 ACC/AHA Guideline on the Treatment of Blood Cholesterol to Reduce Atherosclerotic Cardiovascular Risk in Adults. J Am Coll Cardiol. 2014;63(25_PA):. doi:10.1016/j. jacc.2013.11.002.

Correspondencia: Patricia Lister-Del Pino Dirección: Alameda San Marcos, cuadra 2. Chorrillos, Lima.

Correoelectrónico:patty_lp89@hotmail.com Teléfono: 2477459 - 949559236

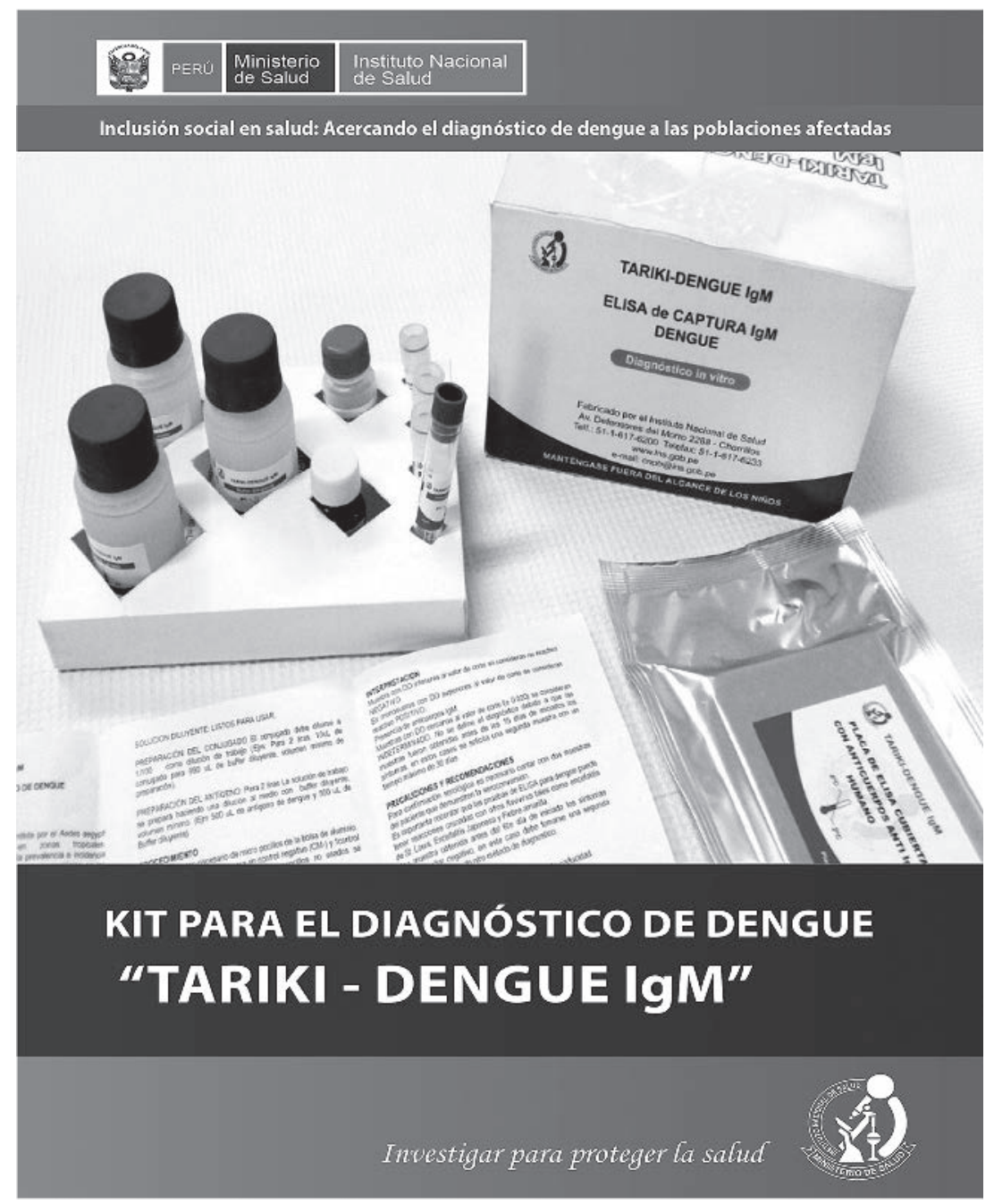

